\title{
Validity of the Actical Accelerometer Step-Count Function in Children
}

\author{
Richard R. Rosenkranz \\ Kansas State University, University of Western Sydney \\ Sara K. Rosenkranz \\ Kansas State University, University of Western Sydney \\ Casey Weber \\ Kansas State University
}

\begin{abstract}
This study sought to assess criterion validity of the Actical monitor step-count function in children via ankle and waist placement, compared with observed video recordings. Children attending a summer program (12 boys, 7 girls, mean age $=9.6 y r s$, range 7-11yrs) wore two synchronized Acticals, attached at the ankle (AA) and waist (AW). Children performed treadmill walking at varying speeds, and two research assistants counted steps using observed video recordings (OVR). Results showed high correlations for AW-OVR $(r=.927, p<.001)$ and AA-OVR $(r=.854, p<.001)$, but AW and AA were significantly lower than OVR $(t>$ $11.2, p<.001)$. AW provided better step estimates than AA for step rates above 130 steps per minute. In contrast, AA was superior to AW for slow walking, and measured more steps during the (nontreadmill) program time. Overall, the Actical monitor showed good evidence of validity as a measure of steps in children for population-based studies.
\end{abstract}

In the wake of a well-documented epidemic of childhood overweight and obesity $(2,7,14)$, there has emerged a strong interest in the physical activity levels of children and adolescents (17). Physical activity (PA), particularly PA done at a moderate-to-vigorous level (MVPA), has been shown to be a protective factor for overweight and obesity $(13,16)$. The health benefits of PA for children and adolescents, however, extend well beyond avoidance of weight-related problems. For example, there is accumulating evidence for the beneficial effects of PA on musculoskeletal health, cardiovascular health, blood pressure, blood lipids, selfconcept, anxiety, depression, and academic performance (16). Therefore, the level of children's PA participation is a prominent public health concern.

R. Rosenkranz and S. Rosenkranz are with Kansas State University, Manhattan, KS, and University of Western Sydney, Campbelltown, NSW, Australia. Weber is with Kansas State University, Manhattan, KS. 
There is an ongoing need for the development of convenient and accurate measures of PA for population-level surveillance of child PA levels and for health outcomes research. In recent years, there has been ample interest in determining the relationships between sedentary behavior, PA of varying intensities, and numerous health outcomes, including obesity. Public health messages have focused on children achieving a recommended 60 min of MVPA daily (16), but the measurement tools and approaches used to assess the achievement of this standard continue to present difficulties. The use of accelerometer-based objective monitors has simultaneously solved problems with regard to eliminating self-report biases, and created new problems regarding processing and interpreting the accelerometer count data (12). Research is ongoing toward building consensus on interpretation of accelerometer count data from various accelerometer-based monitors. Due to these difficulties in characterizing PA intensity level, many researchers have focused on overall levels of PA, frequently measured as daily steps $(4,10,19)$.

Dual-function accelerometers have recently been introduced to measure physical activity objectively in both accelerometer counts and step counts simultaneously (18). An advantage of using dual-function accelerometers rather than pedometers is the ability to link step count data with time of day, as dual-function accelerometer data are time stamped. Such time stamping then adds to the measure of total accumulated physical activity by allowing researchers to ascertain patterns of physical activity and physical inactivity over time. The Actical activity monitor is one such dual-function PA measurement device that is small, water-resistant, and designed to be worn at the waist, wrist, or ankle (8). Previous studies have validated the Actical monitor in both children and adults as a measure of activity energy expenditure within light, moderate, and vigorous intensities (8). Other evidence shows that the Actical can provide valid step-count estimates using waist placement of the device in a diverse population (6). Our review of the literature, however, failed to show any validity assessment of the Actical step-count function in a strictly pediatric sample, or for placement areas besides the waist. Therefore, this study sought to assess the criterion validity of the Actical step-count function in children using ankle and waist placement in comparison with observed video recordings, during a structured treadmill continuous ramp protocol. Secondarily, we assessed the concurrent validity of ankle-placed Actical monitors by comparing them to a previously validated pedometer in a structured protocol, and to waistplaced Actical monitors in more free-living conditions. Due to difficulties that are well established in the literature for measuring steps during slow walking $(5,6)$, we hypothesized that the ankle-placed Actical would provide a more valid measure of steps than the waist-placed monitor, stemming from the relatively greater force oscillations at the ankle (8).

\section{Methods}

\section{Participants}

Our sample consisted of a diverse group (10 non-Hispanic Caucasian, 7 Asian, 1 African-American, 1 Hispanic) of able-bodied children attending a summer program ( 12 boys, 7 girls, mean age $=9.6 y r s$, range $7-11 \mathrm{yrs}$ ) (see Table 1). Parents were informed of research procedures, and gave informed written consent for 
Table 1 Summary of Participant Characteristics in the Sample $(n=19)$

\begin{tabular}{lcc}
\hline & Mean $\pm S D$ & Range \\
\hline Age $(\mathrm{yr})$ & $9.6 \pm 1.2$ & $7.3-11.8$ \\
Height $(\mathrm{cm})$ & $138.1 \pm 10.9$ & $119.2-157.7$ \\
Body Mass $(\mathrm{Kg})$ & $34.2 \pm 10.4$ & $21.0-53.7$ \\
BMI & $17.6 \pm 3.2$ & $14.7-24.2$ \\
BMI Percentile & $53.1 \pm 26.9$ & $5-98$ \\
\hline
\end{tabular}

participation of children before the start of the study. Children gave verbal assent before all measurements and activities. Our study was approved by the IRB at Kansas State University.

\section{Procedure}

Children took part in one or both weeks of a summer four-hour program entitled "Finding a Healthy Rhythm" with a focus on fun physical activities, music, games, healthful snacks, arts and crafts. This summer program was promoted to local parents via newsletters, emails, and postings at the university, and was billed as a "Last chance for summer fun before school starts again." The program began each day at 8:00am, and ended at noon. Typically, the day began with a half-hour of free-play activities in a well-equipped gymnasium. Next, a series of nonelimination games and activities followed for about 30-45 min, accompanied by high-energy music. After that, a fifteen-minute drink and bathroom break followed. Less physically active "team-building" cooperative challenges then followed for about $30 \mathrm{~min}$. Next, children relocated to a classroom for a brief nutrition education class, followed by hands-on healthful snack creation and consumption. After snack and clean-up, children did arts and crafts or classroom games for 15-30 min. The remainder of camp time was then spent outside playing active games or enjoying other activities until pick-up time by a parent.

All camp children individually participated in our research study by wearing activity monitors for up to four hours during one camp day, including a 15-min structured treadmill continuous ramp protocol (see details below). At program day's beginning, children were fitted with two Actical monitors, and before the treadmill portion of the study, each child was fitted with a pedometer (details below).

\section{Accelerometer-Based Monitor}

We used four pairs (eight total) Actical activity monitors (Respironics Inc. Murrysville, PA), that had been used previously by our research team and known to be in good working order, and to provide face-valid data from observations of physical activity sessions in girl scouts. Actical monitors are small $(1.14 \times 1.45$ $\times 0.43 \mathrm{in}$ ), lightweight $(0.56 \mathrm{oz})$, waterproof devices that are powered by lithium coin cells and able to measure movement across a range of forces $(0.05 \mathrm{G}$ to 2.0 $\mathrm{G})$ at a sampling rate of $32 \mathrm{~Hz}$. Actical monitors can be programmed to record 
accelerometer count data in epochs ranging from 15 to $60 \mathrm{~s}$, with a maximum data storage capacity of 11-44 days. Previous research has shown the intra- and interinstrument variability of the Actical to be small (6) and we maintained consistency of comparisons by assigning each participant one of four pairs of monitors to be worn at the waist and ankle simultaneously. Waist placement was accomplished with an adjustable elastic band, fitting the monitor on the right anterior iliac spine. Ankle placement was accomplished with a disposable nylon locking band, fitting the monitor just proximal to the lateral malleolus. A wristwatch, analog clock, and each set of monitors was synchronized with the initializing computer, and monitors were programmed to record count data in $30 \mathrm{~s}$ epochs beginning at 8:00am on the study day. A research assistant recorded the time when each numbered monitor was placed on a participant, as well as when the monitor was removed, usually at the end of the program day, just before noon. Wear time was assessed first by careful research assistant supervision of participating children, and confirmed via post hoc visual observation of subject data. From all children's wear time, we determined that nonwear time was limited to program late arrivals and early departures. In all cases, the participants wore both Actical monitors at least 3.5 continuous hours, and for the full duration of the treadmill continuous ramp protocol portion.

\section{Pedometer}

We used one Yamax Digiwalker SW-200 pedometer (Yamax Corp., Japan), assessed for accuracy before monitoring, for all participants. The Digiwalker pedometer is a small $(2.0 \times 1.5 \times 0.75 \mathrm{in})$, lightweight $(<0.25 \mathrm{oz})$, inexpensive $(\sim 25 \mathrm{US})$, spring-levered step counter with a sensitivity threshold of $0.35 \mathrm{G}$. This pedometer has demonstrated good reliability and validity as a measure of steps and overall physical activity in a range of populations, including children $(1,9)$. Before the treadmill continuous ramp protocol, the Digiwalker pedometer step count was reset to zero and the pedometer was placed on the same elastic waist band shared by the Actical, with placement on the left anterior iliac spine. After completion of the treadmill continuous ramp protocol, the pedometer was carefully removed, cover opened, and step count recorded manually by a research assistant.

Treadmill Continuous Ramp Protocol One Quinton treadmill (Quinton ClubTrack 3.0, Cardiac Science, Bothell, WA), hand-calibrated for determination of speed, was set up for all criterion validity trials. Children were taken individually from the summer program group to complete the 15-min structured treadmill continuous ramp protocol portion of the study. A digital mini-DV video camcorder (Canon ZR-70, Canon USA Inc., Lake Success, NY) was used to record the legs and feet of children while on the treadmill for later observation of step counts. A dry-erase board that indicated participant identity and an analog clock that was synchronized with the Actical monitors and researcher wristwatch were included in the videotaped screenshot for each participant. The continuous protocol began with at least $60 \mathrm{~s}$ of standing still, with the child's hands holding onto the handrails, and feet straddling the belt. At the end of the standing portion, the belt was started and allowed to reach a setting of $1.5 \mathrm{mph}(42 \mathrm{~m} / \mathrm{min})$ maintaining zero gradient of incline. Children were then prompted to step onto the treadmill at the beginning of a $30 \mathrm{~s}$ Actical epoch, with research assistants noting the start time for this stage and all subsequent three-minute stages of the protocol. Each stage was exactly three 
minutes in duration at a constant zero percent grade, and participants remained on the treadmill with no breaks as speeds increased at the beginning of each new stage. Treadmill speeds for the four ambulatory stages were set at $1.5 \mathrm{mph}(42 \mathrm{~m} /$ $\mathrm{min}), 3.0 \mathrm{mph}(83 \mathrm{~m} / \mathrm{min}), 4.5 \mathrm{mph}(125 \mathrm{~m} / \mathrm{min})$, and $6.0 \mathrm{mph}(167 \mathrm{~m} / \mathrm{min})$. To ensure comfort and safety, children were allowed to hold onto the hand rails for balance, if desired, and were allowed to self-select the point at which they transitioned between walking and running. Most children began running in the $125 \mathrm{~m} / \mathrm{min}$ stage, and all children ran in the $167 \mathrm{~m} / \mathrm{min}$ stage. Upon completion of the final stage, children remained on the treadmill belt while a research assistant slowed the treadmill to a full stop within approximately 20-25 s. Children then remained standing still on the belt for an additional minute before moving off the treadmill.

Two trained research assistants later viewed videotapes independently and used a hand tally counter to determine observed video recording (OVR) steps within 30 -s epochs. These research assistants achieved excellent interrater reliability $(r$ $=.998, p<.001)$.

\section{Analyses}

Actical monitor data were downloaded for storage, and matched by participant ID, day, and time in a customized MS Excel 2007 spreadsheet. Pedometer step counts and OVR step counts were hand-entered into a subsection of this spreadsheet, and double-checked for accuracy.

Criterion validity was assessed using Pearson correlations, paired $t$ tests, and Bland-Altman plots (3), all comparing step-count data from ankle-placed Actical monitors and waist-placed Actical monitors to step-count data obtained from the OVR criterion. Due to apparent relationships between difference and magnitude in the Bland-Altman plotting of data, we followed suggestions by Bland and Altman (3) for a logarithmic transformation of each compared measure. This transformation was ineffective at removing nonuniform differences for the creation of $95 \%$ limits of agreement, so we proceeded to model the variability in the standard deviation of differences as a function of measurement level, based on Bland and Altman's suggested method of using absolute residuals from a fitted regression line (3). This rendered V-shaped 95\% limits of agreement for both waist and ankle Actical placement.

Additional concurrent validity was assessed using Pearson correlations and paired $t$ tests to compare step data from ankle-placed Actical and waist-placed Actical over the full program day, and by comparing percentages of OVR steps measured at ankle, waist, and by pedometer. Pearson correlations were used to assess the relationship between child height and difference between criterion and accelerometer/pedometer-measured steps. Microsoft Excel 2007 was used for data compilation, descriptive statistics, and Bland-Altman plots. SPSS (SPSS Inc. Chicago, IL) version 18.0 was used for paired $t$ tests and Pearson correlations. For all analyses, alpha was set at 0.05 .

\section{Results}

For criterion validity tests, there were high correlations between waist-placed Actical and the OVR criterion $(r=.927, p<.001)$ and between ankle-placed Actical and OVR $(r=.854, p<.001)$. Both Actical step estimates were significantly lower than 
OVR step counts $(t>11.2, p<.001)$. Figures 1 and 2 display Bland-Altman plots depicting agreement level between OVR and Actical-derived estimates of steps, with $95 \%$ limits of agreement. Figure 1 shows low agreement at the lower range of steps per minute for waist-placed Actical monitors, relative to good agreement at higher step rates. Figure 2 shows relatively low agreement at the higher range of steps per minute (but within $95 \%$ limits of agreement) for ankle-placed Actical monitors, relative to better agreement at lower step rates.

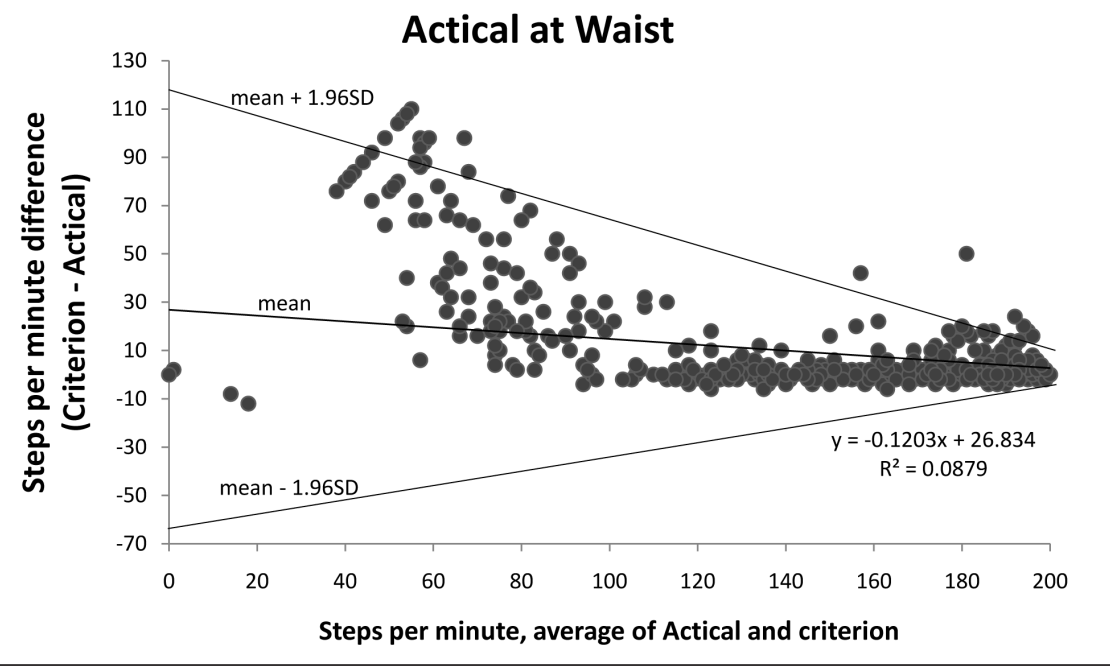

Figure 1 - Bland-Altman plot of agreement between Actical accelerometer placed at the waist and observed video recording criterion measure.

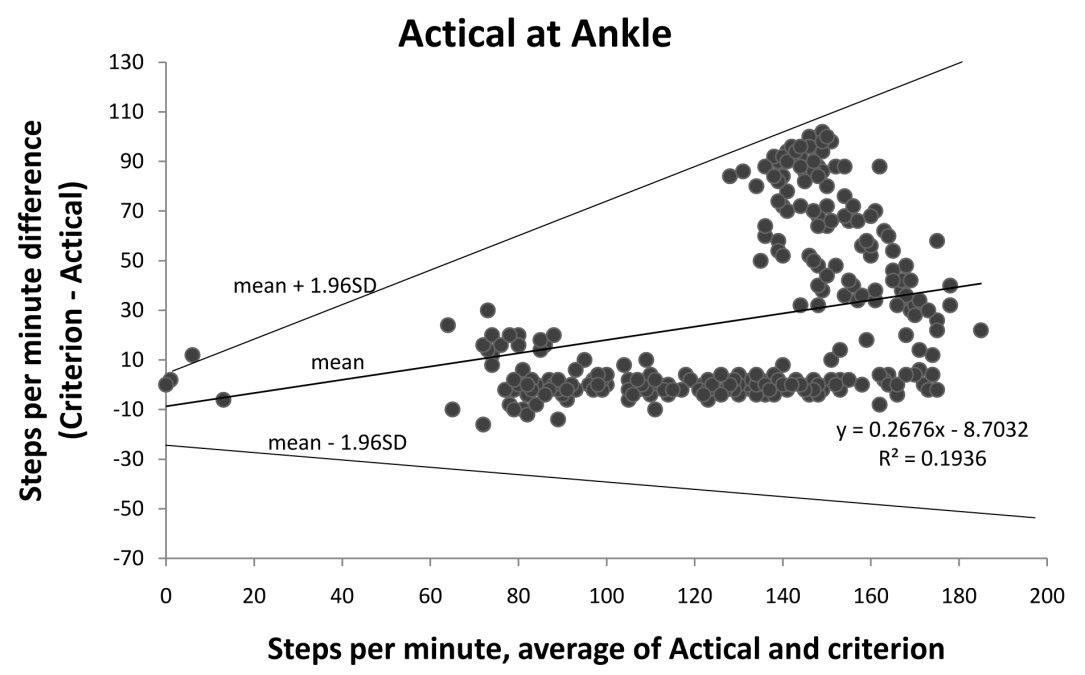

Figure 2 - Bland-Altman plot of agreement between Actical accelerometer placed at the ankle and observed video recording criterion measure. 
For additional concurrent validity tests, there were high correlations between ankle- and waist-placed Acticals $(r=.902, p<.001)$ over the full camp day, but ankle-placement (mean $=51.2$ steps per minute) was significantly higher than waist-placement $($ mean $=45.6$ steps per minute, $t=24.4, p<.001$ ).

Table 2 displays step counts as measured by OVR, Actical monitor, and Yamax Digiwalker SW-200 pedometer. Compared with the OVR criterion measure, waistplaced Actical monitors captured $89 \%$ of treadmill steps, while ankle-placed Actical monitors captured 83\%, and Yamax Digiwalker captured 96\% of treadmill steps. Paired $t$ tests showed that mean step counts from ankle-placed $(t=13.7, p<.001)$ and waist-placed $(t=11.7, p<.001)$ Actical monitors were significantly lower than OVR mean step counts. Total treadmill step counts were not significantly different between Actical waist placement and ankle placement $(t=1.93, p=.07)$, but both were significantly lower than Digiwalker (waist $t=5.5, p<.001$; ankle $t=4.5, p$ $<$.001) total step count.

Table 2 Total Steps Taken During Treadmill Protocol and Camp Day by Measurement Method

\begin{tabular}{|c|c|c|c|c|c|c|}
\hline Child & $\begin{array}{l}\text { Treadmill } \\
\text { Observed } \\
\text { video } \\
\text { recording }\end{array}$ & $\begin{array}{c}\text { Treadmill } \\
\text { Actical- } \\
\text { Waist }\end{array}$ & $\begin{array}{c}\text { Treadmill } \\
\text { Actical- } \\
\text { Ankle }\end{array}$ & $\begin{array}{l}\text { Treadmill } \\
\text { Yamax } \\
\text { Digiwalker }\end{array}$ & $\begin{array}{c}\text { Camp day } \\
\text { Actical- } \\
\text { Waist }\end{array}$ & $\begin{array}{c}\text { Camp day } \\
\text { Actical- } \\
\text { Ankle }\end{array}$ \\
\hline 1 & 1,995 & 1,800 & 1,363 & 1,988 & 9,903 & 12,034 \\
\hline 2 & 1,716 & 1,482 & 1,459 & 1,655 & 7,732 & 9,555 \\
\hline 3 & 1,622 & 1,403 & 1,581 & 1,574 & 6,337 & 7,485 \\
\hline 4 & 1,678 & 1,537 & 1,527 & 1,554 & 9,162 & 11,287 \\
\hline 5 & 1,992 & 1,615 & $\#$ & 1,842 & 7,572 & $\#$ \\
\hline 6 & 1,909 & 1,626 & 1,376 & 1,853 & 10,983 & 12,810 \\
\hline 7 & 1,635 & 1,632 & 1,525 & 1,637 & 14,812 & 13,551 \\
\hline 8 & 1,557 & 1,515 & 1,417 & 1,527 & 9,722 & 11,135 \\
\hline 9 & 1,793 & 1,661 & 1,296 & 1,755 & 8,838 & 9,846 \\
\hline 10 & 1,762 & 1,379 & 1,609 & 1,581 & 6,794 & 10,130 \\
\hline 11 & 1,670 & 1,418 & 1,412 & 1,567 & 7,362 & 8,868 \\
\hline 12 & 1,765 & 1,688 & 1,303 & 1,713 & 12,113 & 9,760 \\
\hline 13 & 1,855 & 1,532 & 1,721 & 1,751 & 11,993 & 15,994 \\
\hline 14 & 1,852 & 1,476 & 1,648 & 1,789 & 12,130 & 14,605 \\
\hline 15 & 1,651 & 1,497 & 1,435 & 1,583 & 11,167 & 15,096 \\
\hline 16 & 1,528 & 1,320 & 1,362 & 1,400 & 10,586 & 11,986 \\
\hline 17 & 1,757 & 1,690 & 1,268 & 1,706 & 12,560 & 11,592 \\
\hline 18 & 1,644 & 1,569 & 1,152 & 1,613 & 12,429 & 10,559 \\
\hline 19 & 1,891 & 1,682 & 1,591 & 1,710 & 12,900 & 14,141 \\
\hline Mean & 1,751 & 1,554 & 1,447 & 1,674 & 10,268 & 11,691 \\
\hline$S D$ & 137 & 125 & 149 & 138 & 2,382 & 2,315 \\
\hline
\end{tabular}

Note. \#ankle data from this participant were not obtained due to programming error. 
We also assessed the relationship between child body height and difference between Yamax Digiwalker and criterion step count, and the relationship between Actical step count and the criterion step count for both waist and ankle placement. There was a significant correlation between height and the difference between waistplaced Actical and criterion $(r=-0.347, p<.05)$, and also between ankle-placed Actical and criterion step count $(r=-0.601, p<.05)$. There was no significant correlation between height and the difference between Yamax Digiwalker and the criterion step count $(r=-0.017, p>.05)$.

\section{Discussion}

This study consisted of a practical assessment of the step-count function of the Actical accelerometer-based monitor, using both ankle placement and waist placement of the monitor with a sample of diverse children. Within extant instrument validation studies, concerns have been raised about the proper approach of protocols to be used. Studies employing treadmills or paced walking on tracks are useful in being able to compare instrument measurements to visual observations under carefully controlled conditions of steady ambulation. Such carefully controlled conditions may bear little resemblance to the real-world conditions of a child's daily physical activities. Employing protocols resembling more real-world conditions in a validation study, however, can be problematic in general, and specifically with regard to researchers' abilities to use visual observation as a criterion variable. In the current study, we blended approaches by using both controlled conditions and real-world protocols. With our highly structured treadmill protocol that used a full range of speeds from standing to running, we were able to test the Actical's ability to measure steps at two placement sites during ambulation, compared with a previously validated pedometer and with an observed video recording criterion. With our analysis of less-structured camp time, we were to compare the ankle-placed step count method to a previously validated waist-placed method.

In the structured treadmill portion of our study, step counts from both ankle placement and waist placement of the Actical correlated highly with our criterion variable of observed video recording step counts. In the more free-living portion of our study, ankle placement correlated highly with the previously validated waist placement of the Actical monitor. Correlation, however, does not constitute agreement, and both placement sites of the Actical monitor provided step counts on the treadmill that were lower than either observed video recordings or a previously validated pedometer.

From analysis of the Bland-Altman plots, it appears that the Actical monitor had difficulty recording children's steps accurately at certain ranges of steps per minute. Based on our treadmill continuous ramp protocol portion of the study, placing Actical either at the ankle or the waist may underestimate step counts compared with validated pedometers or observed video recordings. On the treadmill, waist-placed Acticals provided valid step estimates for ambulatory speeds above $83 \mathrm{~m} / \mathrm{min}$ and at step rates in the 100-200 per min range. Ankle-placed Acticals appeared to provide valid step estimates during slow ambulation, at step rates below 130 per min. Ankle monitoring measured more steps than waist-placed monitoring during the (nontreadmill) camp time, when step rates were usually (>94\% of time) lower than 130 per min. 
Furthermore, we examined the relationship between child body height and differences between Actical step counts and the criterion variable. These analyses indicated that there was a significant relationship, such that greater differences between the Actical step count and criterion were associated with shorter children, and lesser differences were associated with taller children. This influence of height was stronger for ankle placement than it was for waist-placement. A previous study by Stone and colleagues (15) has shown that height/leg length can be influential in accelerometer-based physical activity monitoring. Their study found that accelerometer-derived energy expenditure was under-estimated in taller children, or those with greater leg lengths. In contrast, our results indicate that steps appear to be under-estimated by the Actical in shorter children, for both ankle and waist placement of the Actical.

The present findings support the results of a previous validation study of the Actical's step-count function using waist placement in children and adults (6). One of the most consistent findings in validation studies of pedometers and accelerometers is the difficulty with accurate measurement of slow walking $(5,6)$, and our results showed the same pattern. Similar to the previous Actical step-count function validation study (6), we showed higher error at step counts below 100 per min, but our results add to the literature in that they were obtained from a strictly pediatric sample for the current study. It appears from our findings that the Actical is well suited for capturing step data from a waist placement in children during faster ambulatory activities, in the 100-200 steps per minute range.

Our study breaks new ground in physical activity measurement by testing the validity of the Actical step-count function for placement at the ankle. Although many studies have tested physical activity monitoring devices placed at the ankle $(8,11)$, ours appears to be the first to assess the validity of the Actical step-count function in children. Our findings provide modest support for ankle placement of the Actical. Although it is likely that vigorous-intensity ambulatory activities would be measured with considerable error, less intense ambulatory activities would likely be measured with considerable validity. Thus, it may be most appropriate as a measure of total daily step counts, though it may not be appropriate as a sole measure of physical activity in children with high levels of MVPA. In these populations, total daily step counts could be supplemented with an additional measure of MVPA. The potential importance of the ankle-placement method lies in the utility of the device to be strapped to a child's ankle for multiple days of monitoring without need of removal. Because the Actical is waterproof, it can be secured onto a child's ankle and kept on during bathing, water sports, sleeping, and practically all daily activities. With such a measurement method, there is potential to capture more continuous monitoring time without risk of the child forgetting to remove or put on the monitoring device. The authors have now piloted this method for week-long physical activity monitoring in more than 50 children by using a locking disposable nylon hospital band. With this method, we have encountered few problems (difficulty with soccer shin guards, occasional broken band, minor skin irritation, discomfort when sleeping) and good overall acceptance by children and their parents. The use of a reusable neoprene and Velcro band, available from Actical, would likely improve comfort and prevent problems, but would also sacrifice assurance that the monitor was worn continuously. 
The strengths of the current study include our observed video recordings criterion variable, which consisted of the mean manual step counts derived from two trained observers using videotaped playback. An additional strength of the current study is that we obtained data in both highly controlled and less structured conditions to obtain criterion and concurrent validity data. Other strengths of the study include the novelty of ankle placement assessment, which has potential use in populations who do water-based activities (it may allow children to jump or dive into water without loss of proper positioning) or for those who have difficulty complying with directions to wear waist-placed monitors.

Counterbalancing these strengths are limitations including a relatively small sample size and a lack of true "gold standard" criterion variable for the overall camp time. Future studies should investigate the validity of ankle placement of the Actical in younger children, and undertake further efforts to determine the anthropometric influences such as leg length on the accuracy of step counting.

In conclusion, the Actical monitor appears to provide a valid method for population-based studies to assess physical activity in children via step counts. Use of the Actical for step measurement in pediatric populations would be of benefit in providing data on time-related patterns of physical activity and inactivity. Like most pedometers, waist placement of the Actical appears to have difficulty measuring slow walking, but effectively measures ambulation in the 100-200 steps per minute range. In contrast, ankle placement appears to provide a better measure of slow walking at step rates of less than 130 per minute, but has difficulty measuring steps during fast ambulation.

\section{Acknowledgments}

Funding for this project was provided by a Big XII Faculty Fellowship award at Kansas State University.

\section{References}

1. Barfield, J.P., D.A. Rowe, and T.J. Michael. Interinstrument consistency of the Yamax Digi-Walker pedometer in elementary school-aged children. Meas. Phys. Educ. Exerc. Sci. 8(2):109-116, 2004.

2. Baskin, M.L., J. Ard, F. Franklin, and D.B. Allison. Prevalence of obesity in the United States. Obes. Rev. 6(1):5-7, 2005.

3. Bland, J.M., and D.G. Altman. Measuring agreement in method comparison studies. Stat. Methods Med. Res. 8:135-160, 1999.

4. Cox, M., G. Schofield, N. Greasley, and G.S. Kolt. Pedometer steps in primary schoolaged children: A comparison of school-based and out-of-school activity. J. Sci. Med. Sport. 9(1-2):91-97, 2006.

5. Crouter, S.E., P.L. Schneider, M. Karabulut, and D.R. Bassett, Jr. Validity of 10 electronic pedometers for measuring steps, distance, and energy cost. Med. Sci. Sports Exerc. 35(8): 1455-1460, 2003.

6. Esliger, D.W., A. Probert, S.C. Gorber, S. Bryan, M. Laviolette, and M.S. Tremblay. Validity of the actical accelerometer step-count function. Med. Sci. Sports Exerc. 39(7):1200-1204, 2007.

7. Hedley, A.A., C.L. Ogden, C.L. Johnson, M.D. Carroll, L.R. Curtin, and K.M. Flegal. Prevalence of overweight and obesity among US children, adolescents, and adults, 1999-2002. JAMA. 291(23):2847-2850, 2004.

8. Heil, D.P. Predicting activity energy expenditure using the Actical activity monitor. Res. Q. Exerc. Sport. 77(1):64-80, 2006. 
9. Kilanowski, C.K., A.R. Consalvi, and L.H. Epstein. Validation of an electronic pedometer for measurement of physical activity in children. Pediatr. Exerc. Sci. 11:63-68, 1999.

10. Laurson, K.R., J.C. Eisenmann, G.J. Welk, E.E. Wickel, D.A. Gentile, and D.A. Walsh. Evaluation of youth pedometer-determined physical activity guidelines using receiver operator characteristic curves. Prev. Med. 46(5):419-424, 2008.

11. Louie, L., R. Eston, A. Rowlands, D. Ingledew, and F. Fu. Validity of heart rate, pedometry, and accelerometry for estimating the energy cost of activity in Hong Kong Chinese boys. Pediatr. Exerc. Sci. 11:229-239, 1999.

12. McClain, J.J., T.L. Abraham, and T.A. Brusseau. J.R., C. Tudor-Locke. Epoch length and accelerometer outputs in children: Comparison to direct observation. Med. Sci. Sports Exerc. 40(12):2080-2087, 2008.

13. Ness, A.R., S.D. Leary, C. Mattocks, et al. Objectively measured physical activity and fat mass in a large cohort of children. PLoS Med. 4(3):e97, 2007.

14. Ogden, C.L., M.D. Carroll, and K.M. Flegal. High body mass index for age among US children and adolescents, 2003-2006. JAMA. 299(20):2401-2405, 2008.

15. Stone, M.R., D.W. Esliger, and M.S. Tremblay. Comparative validity assessment of five activity monitors: does being a child matter? Pediatr. Exerc. Sci. 19(3):291-309, 2007.

16. Strong, W.B., R.M. Malina, C.J. Blimkie, et al. Evidence based physical activity for school-age youth. J. Pediatr. 146(6):732-737, 2005.

17. Troiano, R.P., D. Berrigan, K.W. Dodd, L.C. Masse, T. Tilert, and M. McDowell. Physical activity in the United States measured by accelerometer. Med. Sci. Sports Exerc. 40(1):181-188, 2008.

18. Tudor-Locke, C., W.D. Johnson, and P.T. Katzmarzyk. Accelerometer-Determined Steps per Day in US Adults. Med. Sci. Sports Exerc. 41(7):1384-1391, 2009.

19. Tudor-Locke, C., S.M. Lee, C.F. Morgan, A. Beighle, and R.P. Pangrazi. Children's pedometer-determined physical activity during the segmented school day. Med. Sci. Sports Exerc. 38(10):1732-1738, 2006. 